\title{
BMJ Open The acceptance of hearing disability among adults experiencing hearing difficulties: a cross-sectional study
}

\author{
Vinaya K C Manchaiah,, ${ }^{1,2}$ Peter Molander, ${ }^{2}$ Jerker Rönnberg, ${ }^{2}$ \\ Gerhard Andersson, ${ }^{2,3}$ Thomas Lunner ${ }^{2,4}$
}

To cite: C Manchaiah VK, Molander P, Rönnberg J, et al. The acceptance of hearing disability among adults experiencing hearing difficulties: a cross-sectional study. BMJ Open 2014;4: e004066. doi:10.1136/ bmjopen-2013-004066

- Prepublication history and additional material for this paper is available online. To view these files please visit the journal online (http://dx.doi.org/10.1136/ bmjopen-2013-004066).

Received 19 September 2013 Revised 23 November 2013 Accepted 25 November 2013

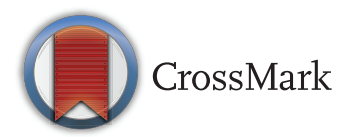

${ }^{1}$ Department of Vision and Hearing Sciences, Anglia Ruskin University, Cambridge, UK

${ }^{2}$ Department of Behavioural Sciences and Learning, Linnaeus Centre HEAD, Swedish Institute for Disability Research, Linköping University, Linköping, Sweden ${ }^{3}$ Division of Psychiatry, Department of Clinical Neuroscience, Karolinska Institutet, Stockholm, Sweden

${ }^{4}$ Eriksholm Research Centre, Snekkersten, Denmark

Correspondence to: Dr Vinaya K C Manchaiah; vinaya.manchaiah@anglia.ac.uk

\section{ABSTRACT}

Objective: This study developed the Hearing Disability Acceptance Questionnaire (HDAQ) and tested its construct and concurrent validities.

Design: Cross-sectional.

Participants: A total of 90 participants who were experiencing hearing difficulties were recruited in the UK.

Outcome measures: The HDAQ was developed based on the Tinnitus Acceptance Questionnaire (TAQ). Participants completed self-report measures regarding hearing disability acceptance, hearing disability, symptoms of anxiety and depression and a measure of stages of change.

Results: The HDAQ has a two-factor structure that explains $75.69 \%$ of its variance. The factors identified were activity engagement and avoidance and suppression. The scale showed a sufficient internal consistency (Cronbach's $\alpha=0.86$ ). The HDAQ also had acceptable concurrent validity with regard to selfreported hearing disability, self-reported anxiety and depression and readiness to change measures.

Conclusions: Acceptance is likely an important aspect of coping with chronic health conditions. To our knowledge, no previously published and validated scale measures the acceptance of hearing disability;

therefore, the HDAQ might be useful in future research. However, the role of acceptance in adjusting to hearing disability must be further investigated.

\section{INTRODUCTION}

An individual with hearing loss might pass through several stages when seeking help. ${ }^{1}$ Edgett ${ }^{2}$ emphasised that the decision-making process to seek help involves four major stages: (1) understanding hearing loss, (2) personal experience, (3) interactions with society and (4) taking action. In another study, Engelund ${ }^{3}$ suggested that patients proceed through four major stages while making a decision to seek help: (1) attracting attention, (2) becoming suspicious, (3) sensing tribulation and (4) jeopardising the fundamental self. In our previous studies of

\section{Strengths and limitations of this study}

- Owing to its online recruitment, the sample might not represent the general population.

- Online questionnaires might differ from a penand-paper format in terms of data quality.

- Although there are measures that study coping indirectly, no well-established acceptance scale to examine the concurrent validity of the Hearing Disability Acceptance Questionnaire using the same construct (ie, acceptance).

the 'patient's journey' regarding people with hearing impairment (PHI), we took this idea further and studied this journey from the initial onset of problems through successful rehabilitation, thereby developing a patient journey model. ${ }^{4} 5$ This model suggests that PHI experience seven major stages before, during and after their audiological rehabilitation: (1) preawareness, (2) awareness, (3) movement, (4) diagnostics, (5) rehabilitation, (6) self-evaluation and (7) resolution. On an average, PHI can take 10 years or more to seek help after first noticing hearing difficulties; hence, this process might take many years. ${ }^{6}$ Although additional research is necessary to better understand this process, ${ }^{7}$ these previous studies nevertheless provide an insight regarding the stages of adjusting to hearing loss, which might be an indirect (or secondary) indicator of the process of acceptance. In addition, it is clear from the aforementioned studies that becoming aware of and accepting a hearing disability play important roles in further progressing in their journey to manage their condition.

A few studies in the audiology literature have focused on the self-assessment of hearing loss, ${ }^{8}$ immediate reactions to the diagnosis of hearing loss, ${ }^{9}{ }^{10}$ actions taken after failing screening tests, ${ }^{11}$ attitudes towards hearing loss and the use of hearing 
aids ${ }^{12} 13$ and the ways in which PHI cope with their condition. ${ }^{14-16}$ These studies provide further insights into the initial onset of the condition and how PHI manage their condition. A recent literature review suggested that the acceptance of hearing loss prior to hearing aid fitting positively influences hearing aid acquisition and subsequent hearing aid use. ${ }^{17}$ However, numerous PHI who are aware that they have a problem but continue not to seek help or use intervention strategies, or both remain. The literature can be confusing because the term 'acceptance' is used synonymously with the treatment/management adherence, rather than studying this construct using validated scales that capture different dimensions of this construct.

In psychological terms, acceptance is a process of actively taking in thoughts, memories, feelings and bodily sensations in a specific situation without having to follow or change them. ${ }^{18}$ Acceptance can have various dimensions including self-acceptance and social acceptance that require the emotional and behavioural adaptations. For instance, the different views of what acceptance actually involves are evident from examining studies of education and academic success, where the term peer acceptance refers to a pupil who is judged to be a desirable interaction partner. ${ }^{19}$ Disability studies have proposed that acceptance is the key component to adjusting to a disabling condition. ${ }^{20}$ Although the acceptance of chronic conditions has often been studied from the perspectives of grief and loss, studying the acceptance of chronic conditions with regard to the perspectives of those living with it, with a focus on adaptation to and accepting change in one's life, is also important. ${ }^{21}$

Experiential avoidance is similar to the concept of avoidance coping and can be defined as the opposite reaction to acceptance; that is, a person attempts to ignore and minimise the problems caused by hearing impairment. ${ }^{22}$ However, some researchers have argued that coping and experiential avoidance are unique but overlapping constructs. ${ }^{23}$ For example, although acceptance (ie, experiential avoidance) loaded onto the same factors as emotion-focused and avoidant coping in a recent study of anxiety disorders, acceptance explained additional variance when predicting psychological distress and well-being. ${ }^{23}$ With regard to hearing impairment, acceptance and experiential avoidance can be interpreted in light of stigma theory, ${ }^{24}$ which has previously been applied in hearing impairment research. ${ }^{25} 26$

Acceptance, in relation to hearing disability, has not been well defined; often this term is used to refer the help-seeking behaviour and intervention (eg, hearing aids) adoption. In effect, differences exist in terms of the psychological and audiological ways of defining acceptance with regard to hearing disability. However, various scales examine the acceptance of other disabilities and chronic conditions. ${ }^{20} 21{ }^{27-30}$ Past audiology studies have focused on coping; however, coping is generally measured indirectly via questions related to communication problems ${ }^{22}$ (eg, the Communication
Strategies Subscale (CSS) in the Communication Profile for the Hearing Impaired (CPHI) questionnaire). ${ }^{31}$ The CPHI-CSS focuses on maladaptive behaviours as well as verbal strategies and non-verbal strategies, and it provides insight concerning poor adjustment to hearing impairment and poor social support. ${ }^{32}$ To our knowledge, however, no published and validated scale examines the acceptance of hearing disability.

The current study developed a self-report measure of hearing disability acceptance and investigated its construct and concurrent validities. We focused on the psychological aspects of acceptance in this study (ie, experiential avoidance, which is the opposite of acceptance).

\section{METHOD}

\section{Study design and participants}

The current study used cross-sectional data obtained during a clinical trial (ie, preintervention data) of a prefitting counselling programme. ${ }^{33}{ }^{34}$ A study advertisement was made in the UK through various sources including national newspapers, hearing loss charity websites (ie, Action on Hearing and Hearing Link) and local general practitioner practice notice boards, inviting those who were experiencing hearing difficulties but not using hearing aids and also those who had access to the Internet to participate in this study. Interested participants were encouraged to access the study website using the URL supplied. A total of 90 participants completed the informed consent form, provided demographic information and completed four online questionnaires. These questionnaires included the Hearing Disability Acceptance Questionnaire (HDAQ), the Hearing Handicap Questionnaire (HHQ), the Hospital Anxiety and Depression Scale (HADS) and the University of Rhode Island Change Assessment (URICA) Scale.

\section{Development of the HDAQ}

The HDAQ was developed based on the Tinnitus Acceptance Questionnaire (TAQ), which was developed in Sweden to study tinnitus acceptance. ${ }^{30}$ The TAQ was based on the Acceptance and Action Questionnaire $(\mathrm{AAQ})^{35}$ and the Chronic Pain Acceptance Questionnaire-Revised (CPAQ-R $)^{28}$; some additional questions were included. The 12-item TAQ has two factors (activity engagement and tinnitus suppression), and it has sufficient internal consistency (Cronbach's $\alpha=$ 0.89) ${ }^{30}$ Like the TAQ, the HDAQ is a measure of experiential avoidance/acceptance. Its 12 items were taken from the TAQ and the word 'tinnitus' was replaced with 'hearing problem'. However, the 12 items were further reduced to 7 items (see the results section). Each item was rated on a 7-point Likert scale (1=never true, $7=$ always true). Total scores ranged from 7 to 49 ; higher scores indicate greater acceptance of hearing disability (see online supplementary appendix 1 ). 


\section{Other questionnaires}

The HHQ measures personal and social effects (ie, emotional distress and discomfort, social withdrawal and general participation restrictions). ${ }^{36}$ The 12 questions of the HHQ are scored on a 5-point Likert scale ( $1=$ never, $5=$ almost always). Total scores range from 12 to 60 , and higher scores indicate a greater disability. The HHQ has acceptable internal consistency, with Cronbach's $\alpha$ of 0.95 and 0.93 for the emotional and social scales, respectively.

The HADS was used to screen for symptoms of anxiety and depression. The HADS consists of 14 items, divided into two subscales: anxiety and depression. ${ }^{37}$ Each item is scored from 0 to 3 ( $0=$ not at all, $3=$ most of the time) with a total score ranging from 0 to 42 ; higher scores indicate more self-reported anxiety and depressive symptoms. The HADS has acceptable reliability $(r=0.84)$ and internal consistency $(\alpha=0.83),{ }^{38}$ including Internet administration. ${ }^{39}$ In addition, the HADS also has acceptable sensitivity and specificity $(\mathrm{AUC}=0.80$ ) as indicated in the receiver operator characteristic curves.

The URICA measures stages of change across four subscales: precontemplation, contemplation, action and maintenance. ${ }^{40}$ The original URICA scale consists of 32 items; however, the current study used a modified version (the problem was replaced with the hearing problem) consisting of a 24-item scale. ${ }^{42}$ Each item was rated on a 5-point Likert scale ( 1 =strong disagreement, $5=$ strong agreement), and each subscale measured specific aspects. Most of the study participants were in the early help-seeking stages and had not received interventions for their hearing disabilities; therefore, the eight URICA items regarding maintenance were excluded because they were considered irrelevant for the sample. The total scores of each subscale ranged from 8 to 40 . The subscale scores concerning the contemplation and action stages were added, from which the precontemplation stage scores were subtracted to obtain a readiness-to-change composite score (ie, contemplation +action-precontemplation). A recent study used this modified scale to investigate the use of the URICA scale among adults with acquired hearing impairments seeking help for the first time. This scale showed acceptable construct, concurrent and predictive validities. ${ }^{42}$

\section{Data analyses}

All data analyses were performed using IBM SPSS V.19 for Windows. Descriptive statistics were applied to examine demographic factors, and the assumption of normality (ie, Shapiro-Wilk test values of 0.05 ) was tested before conducting a principal components analysis (PCA). A PCA was performed to reduce the correlated variables to a smaller set of important composite variables and examine the factor structure ${ }^{43}$ Cronbach's $\alpha$ was calculated to assess the internal consistency of the HDAQ. ${ }^{44}$ Pearson's correlations were performed to examine the association among the following factors: hearing disability acceptance, self-reported hearing disability, self-reported anxiety and depression and readiness to change.

\section{RESULTS}

The data were normally distributed. Table 1 displays the sample characteristics. The average age of participants and the average duration of hearing disability were 63.41 and 11.67 years, respectively. The number of men and women in the sample was equal. In addition, nearly two-thirds of participants had consulted healthcare professionals specialised in hearing (eg, audiologists, hearing aid dispensers or ear, nose and throat specialists) at least once.

\section{HDAQ factor structure}

A PCA with Varimax rotation was performed to examine the factor structure. Eigenvalues were set at 1.0, and the limit for factor loadings was set at $0.40{ }^{43}$ The relevant items were reverse scored before analysis. The initial number of factors of interest was determined using Kaiser's rule of eigenvalues greater than 1.0. ${ }^{45}$ Subsequently, a scree plot was examined to determine the number of factors to extract. ${ }^{46}$

In the first instance, the PCA resulted in a three-factor model for the 12 items. However, cross-loadings were noted for some items (ie, items that loaded at 0.40 or above on two or more factors). A PCA was also performed using Direct Oblimin rotation to determine whether these cross-loadings were due to the high correlations among items. The Kaiser-Meyer-Olkin (KMO) measure of sampling adequacy was 0.86 with a reference value of $0-1.0$, and Bartlett's test of sphericity was significant $\left(\chi^{2}(66)=370.89, \quad \mathrm{p}<0.001\right)$. The three factors explained $72.90 \%$ of the variance in the 12-item HDAQ Factor 1 accounted for $49.65 \%$ of the variance (with an eigenvalue of 3.40 ); factor 2 accounted for $13.95 \%$ of the variance (with an eigenvalue of 3.32) and factor 3 accounted for $9.30 \%$ of the variance (with an eigenvalue of 2.01). The Cronbach's $\alpha$ was $0.54,0.50,0.72$ and 0.79 for factors 1, 2, 3 and the overall 12 items, respectively. The internal consistency of the overall scale was acceptable, although it was not high for factors 1 and 2 .

In the next stage, all items that resulted in crossloadings were removed from the analysis; thus, five items were removed from the original 12-item scale (see online supplementary appendix 2 for the removed items). Item reduction (ie, minimising the set of variables while still accounting for most of the variance) is one of the key goals of PCA. Moreover, removing the items with complex psychometric properties (ie, cross loadings) can improve the construct validity of selfreport measures. ${ }^{47}$ Subsequently, the seven-item HDAQ resulted in a two-factor model with no cross-loading or outliers (see table 2). The KMO measure of sampling adequacy was 0.82 , and Bartlett's test of sphericity was significant $\left(\chi^{2}(21)=363.93, \quad \mathrm{p}<0.001\right)$. These factors explained $75.69 \%$ of the variance in the HDAQ. Factor 
Table 1 Participants demographics

\begin{tabular}{|c|c|}
\hline Age in years $(M \pm S D)$ & $63.41 \pm 10.49$ \\
\hline Gender (\% male) & 50 \\
\hline Duration of hearing difficulties in years $(M \pm S D)$ & $11.67 \pm 10.83$ \\
\hline \multicolumn{2}{|c|}{ Consulted a healthcare professional specialising in hearing regarding hearing difficulties (\%) } \\
\hline Yes & 65.6 \\
\hline No & 34.4 \\
\hline \multicolumn{2}{|l|}{ Education (\%) } \\
\hline Compulsory education & 13.3 \\
\hline Secondary education & 48.9 \\
\hline Tertiary education & 37.8 \\
\hline Self-reported hearing disability acceptance (HDAQ; $M \pm S D)$ & $36.88 \pm 7.85$ \\
\hline Activity engagement & $22.72 \pm 4.36$ \\
\hline Avoidance and suppression & $14.16 \pm 4.65$ \\
\hline Self-reported hearing disability $(\mathrm{HHQ} ; \mathrm{M} \pm \mathrm{SD})$ & $34.96 \pm 9.95$ \\
\hline Emotional & $20.61 \pm 5.75$ \\
\hline Social & $14.32 \pm 4.85$ \\
\hline Self-reported anxiety and depression (HADS; $\mathrm{M} \pm \mathrm{SD}$ ) & $14.77 \pm 7.50$ \\
\hline Anxiety & $7.04 \pm 4.43$ \\
\hline Depression & $7.70 \pm 3.81$ \\
\hline \multicolumn{2}{|l|}{ Stages of change (URICA) } \\
\hline Readiness-to-change composite $(\mathrm{M} \pm \mathrm{SD})$ & $39.41 \pm 8.63$ \\
\hline
\end{tabular}

1 accounted for $42.94 \%$ of the variance (with an eigenvalue of 3.00) and factor 2 accounted for $32.75 \%$ of the variance (with an eigenvalue of 2.29). The factors were identified as: (1) activity engagement (ie, the pursuit of life activities regardless of hearing disability) and (2) avoidance and suppression (ie, attempts to avoid difficult listening situations as well as those to control and suppress the thoughts and feeling related to hearing disability). A low avoidance and suppression score indicates more avoidance and suppression because the items are reverse scored. The Cronbach's $\alpha$ were $0.90,0.82$ and 0.86 for factors 1, 2 and the overall HDAQ, respectively, showing an acceptable internal consistency. ${ }^{44}$ The correlation between the factors was $r(90)=0.51, p<0.001$, which suggests that the two subscales were distinguishable but related.

Furthermore, we performed a PCA on the split sample to test for the generalisability (ie, split-sample validation). The sample of 90 was randomly divided into two groups of 45. The PCA for the first split sample of 45 on 12 items resulted in a three-factor model with cross-loadings. However, the PCA with seven items (after the removal of the items with cross loadings) resulted in a two-factor structure that explained $76.41 \%$ of the variance without cross-loadings. A PCA was also performed on the second-split sample of 45 that also resulted in a

Table 2 HDAQ principle components analysis $(n=90)$

\section{Scale: 7-item HDAQ}

1. I am leading a full life, even though I have a hearing problem

2. My life is going well, even though I have a hearing problem

3. Despite hearing problem, I can draw up and stick to a certain course in my life

4. When my hearing problem increases, I can still take care of my responsibilities

5. My hearing problem leads me to avoid certain situations

6. My hearing problem changes me as a person

7. I spend a lot of time thinking about how things would be for me without a hearing problem

Cronbach's $\alpha$

Percentage of variance

Eigenvalue
Factor 1: activity Factor 2: avoidance and engagement suppression

0.854
0.891
0.857

0.763

0.885

0.862

0.734

0.90

42.94

3.0

0.82

32.75

2.29

HDAQ, Hearing Disability Acceptance Questionnaire. 
two-factor structure that explained $75.93 \%$ of the variance without cross-loadings. The results from the split samples and the total sample were in close agreement (see table 3), strongly supporting the two-factor model.

\section{Correlations between the HDAQ and other scales}

Table 4 shows the Pearson's correlation coefficients between the HDAQ and other scales. The two HDAQ subscales were moderately associated with each other and strongly associated with the full scale. The results revealed the following significant correlations: a moderately strong negative correlation between acceptance and hearing disability; a moderate negative correlation between acceptance and symptoms of anxiety and depression and a weak negative correlation between acceptance and readiness to change. These results suggest that those people with higher hearing disability acceptance had less self-reported hearing disability, fewer self-reported symptoms of anxiety and depression and lower readiness to change. In addition, a weak positive correlation was found between self-reported hearing disability and self-reported symptoms of anxiety and depression, which indicates that people with higher selfreported hearing disability are likely to have higher selfreported symptoms of anxiety and depression. Although differences were observed, the relationships between the individual subscales and other factors (ie, hearing disability, symptoms of anxiety and depression and readiness to change) did not differ much compared with the full HDAQ scale.

\section{DISCUSSION}

Recent research has indicated the utility of psychological acceptance with regard to reducing the impact of chronic health conditions. ${ }^{29}{ }^{48-50}$ The current paper is the first known attempt to extend this concept to hearing disabilities. After the necessary psychometrical modifications, a two-factor structure emerged for the HDAQ that was in line with the $\mathrm{TAQ}^{30}$ and similar selfreport measures related to acceptance. ${ }^{51}$ Its internal consistency was equal to that of the most commonly used general acceptance scale, the AAQ-I. ${ }^{52}$ However, research on an updated version of this scale rejected a two-factor structure with regard to measuring acceptance and suggested a unidimensional structure for the AAQ-II. ${ }^{53}$ Our study did not reveal a single-factor structure for the HDAQ. The first factor, activity engagement, was assumed to reflect whether participants maintain a desired level of activity despite facing obstacles. For example, a low level of activity engagement might be observed in a person who stops dining with his or her friends at restaurants because they fear they might not be able to follow the conversation. The other factor, avoidance and suppression, is the unwillingness to experience events due to their related emotional distress. This concept is the opposite of acceptance. ${ }^{54}$

The HDAQ items were taken from the scale used to study acceptance in people with tinnitus, which, in turn, was based on the AAQ and CPAQ-R. Although differences most likely exist with regard to how people cope with tinnitus and hearing disability, similarities are found in terms of how people cope with general chronic conditions. Acceptance is likely a key component of adjusting to a disabling condition. ${ }^{20}$ Thus, the study of acceptance in various chronic conditions including hearing disability using the general framework applied in 'contextual psychology' is valuable to the researchers and clinicians.

The study results indicate that less acceptance was associated with increased emotional distress. The relationship between the HDAQ and the HADS is interesting when compared with similar studies that have indicated the pivotal role of acceptance with regard to suffering. For instance, McCracken ${ }^{55}$ found that acceptance was a stronger predictor of psychosocial disability among patients with chronic pain than the degree of pain they experienced. Numerous other studies have also found that greater acceptance is related to psychological well-being (for a summary, see the review by Ruiz $^{56}$ ). In the present study, greater acceptance was

Table 3 Split-sample validation for the seven-item Hospital Anxiety and Depression Scale, Hospital Anxiety and Depression Scale

\begin{tabular}{|c|c|c|c|}
\hline & Full sample $(n=90)$ & Split sample $1(n=45)$ & Split sample $2(n=45)$ \\
\hline \multicolumn{4}{|l|}{ Percentage of variance explained } \\
\hline Factor 1: activity engagement & 42.94 & 43.48 & 44.13 \\
\hline Factor 2: avoidance and suppression & 32.75 & 32.93 & 31.80 \\
\hline Combined & 75.69 & 76.41 & 75.93 \\
\hline \multicolumn{4}{|l|}{ Eigenvalue } \\
\hline Factor 1 : activity engagement & 3.0 & 3.0 & 3.0 \\
\hline Factor 2: avoidance and suppression & 2.29 & 2.3 & 2.2 \\
\hline \multicolumn{4}{|l|}{ Cronbach's $\alpha$} \\
\hline Factor 1: activity engagement & 0.90 & 0.83 & 0.82 \\
\hline Factor 2: avoidance and suppression & 0.82 & 0.89 & 0.90 \\
\hline Combined & 0.86 & 0.88 & 0.86 \\
\hline
\end{tabular}




\section{Table 4 Correlations between different scales}

\begin{tabular}{|c|c|c|c|c|c|c|}
\hline & HDAQ & HDAQ-1 & HDAQ-2 & HHQ & HADS & URICA-R \\
\hline Self-reported hearing disability acceptance (HDAQ) & 1.00 & & & & & \\
\hline HDAQ Factor 1: Activity engagement & $0.86^{*}$ & 1.00 & & & & \\
\hline HDAQ Factor 2: Avoidance and suppression & $0.88^{*}$ & $0.51^{*}$ & 1.00 & & & \\
\hline Self-reported hearing disability (HHQ) & $-0.70^{\star}$ & $-0.50^{\star}$ & $-0.71^{\star}$ & 1.00 & & \\
\hline Self-reported anxiety and depression (HADS) & $-0.58^{*}$ & $-0.62^{*}$ & $-0.39^{*}$ & $0.36^{*}$ & 1.00 & \\
\hline Readiness to change (URICA-R) & $-0.27^{*}$ & $-0.26^{*}$ & $-0.29^{*}$ & 0.20 & 0.18 & 1.00 \\
\hline
\end{tabular}

correlated with a more modest rating of hearing problems; therefore, it might be analogous to the aforementioned findings regarding pain. Moreover, a recent systematic review showed that self-reported hearing disability is a robust predictor of hearing help-seeking, hearing aid acquisition, hearing aid use and satisfaction with hearing rehabilitation, ${ }^{17}$ which suggests that selfreported hearing disability is an important factor in the processes of accepting hearing loss and seeking appropriate interventions. Furthermore, symptoms of depression and anxiety were elevated among those who scored higher on the HHQ which illustrates the far-reaching consequences that hearing loss might have. The relationship between the HDAQ and readiness to change was negative and significant, which implies that those who were more accepting of their current status were less prone to seek a change in their current situation. The association between the HDAQ subscales and other factors did not differ much. This finding might be because both subscales were found to have strong associations with the full scale and moderate associations with each other.

Interestingly, although participants did not use hearing aids, two-thirds of the sample had consulted hearing specialists on at least one occasion. The reasons for these consultations are likely to include: (1) acceptance of their condition (ie, hearing disability); (2) a dilemma regarding whether they had the condition and a desire to confirm this supposition with clinicians or (3) urging from their social partners. Although the reasons for not accepting intervention (eg, hearing aids) are not clear, they might be related to the perceived seriousness of their hearing problems. Previous research suggests that a linear relationship does not exist between hearing disability and its effects on activities and participation, ${ }^{8} 57$ which might help explain why not all people with hearing disabilities seek interventions. Thus, studying hearing disability acceptance in psychological terms is important. Importantly, becoming suspicious or aware of a hearing disability does not mean that PHI perceives their difficulties as significant enough to affect their communication and quality of life. This gap between awareness and action is something that clinicians must be aware of when they plan and recommend interventions, especially for patients coming to the hearing clinic for the first time. In addition, much work is needed to understand how hearing disability acceptance either facilitates or hinders the journey through this condition.

\section{Study limitations}

Although the current study focused on an important area of limited research, it nevertheless has certain limitations. Acceptance with regard to hearing disability is not well defined, and this scale might only focus on certain components of acceptance (ie, psychological acceptance). This limitation might partially explain why people with greater acceptance show less readiness to change. However, this component is important to understand because it might explain why many people who are aware of their hearing disability continue to refrain from seeking professional help and appropriate interventions. Owing to the online recruitment method, the sample might not represent the general population, and caution must be used in generalising the results. ${ }^{58} 59$ Moreover, the relatively small sample size was surprising, given that the advertisement was published in a national newspaper. The smaller sample size might also be a limitation of this study. Validating this scale with a larger population is necessary, although the split-sample validation strongly supported the two-factor model. The online format of the questionnaire might differ from a pen-and-paper format, although web-based questionnaires have been found to be reliable and valid. ${ }^{6061}$ The study results are only relevant with regard to participants who experience hearing difficulties rather than typical participants in clinical situations, although there might be some overlap because nearly two-thirds of the current sample had previously consulted hearing specialists. Although indirect coping measures exist, no wellestablished acceptance scale examines the concurrent validity of the HDAQ using acceptance. Studying the associations between acceptance and other factors such as cognitive functions, personality, quality of life and psychological well-being would have been interesting and useful; however, these factors were not included in the current study. Furthermore, the predictive validity of the scale must be explored. 


\section{CONCLUSIONS}

In summary, our results suggest that additional exploration of the potential role that acceptance plays in the process of adjusting to hearing problems would be a fruitful endeavour, particularly with regard to understanding the role that acceptance plays in the journey of PHI. However, much work remains to be performed. Specifically, a coherent theoretical framework is needed to account for what role, if any, acceptance plays with regard to adjusting to hearing problems. One cannot take for granted the fact that the successful management of other conditions, which all entails painful experiences (eg, tinnitus, chronic pain and anxiety), is relevant to hearing disability, which instead is characterised by the loss of (auditory) experiences. In addition, future research must examine the longitudinal stability of acceptance and its relevance to objective measures of hearing disability as well as the utility of the current structure of the HDAQ and whether it can successfully alleviate the suffering usually associated with hearing disability.

Acknowledgements The authors would like to thank Alexander Alasjö for his help with applying the online questionnaires and Ariane Laplante-Lévesque for providing us with the revised version of the URICA scale used in this study. This study was a part of the doctoral study of the first author, and portions of this manuscript were presented as a doctoral thesis.

Contributors VKCM contributed to most of the work including data collection, analysis and writing; PM contributed to the writing; JR contributed to study design and writing; GA contributed to study design and writing; and TL contributed to study design and writing.

Funding Grants from the Swedish Council for Working Life and Social Research 2009-0055 as well as the Swedish Research Council 2007-8654 partially funded this research.

Competing interests None.

Patient consent Obtained.

Ethics approval Ethical approval was received from the Research Ethics Committee, College of Human and Health Sciences, Swansea University.

Provenance and peer review Not commissioned; externally peer reviewed.

Data sharing statement No additional data are available.

Open Access This is an Open Access article distributed in accordance with the Creative Commons Attribution Non Commercial (CC BY-NC 3.0) license, which permits others to distribute, remix, adapt, build upon this work noncommercially, and license their derivative works on different terms, provided the original work is properly cited and the use is non-commercial. See: http:// creativecommons.org/licenses/by-nc/3.0/

\section{REFERENCES}

1. Stephens D, Kramer SE. Living with hearing difficulties: the process of enablement. Chichester: Wiley, 2009.

2. Edgett LMD. Help-seeking for advanced rehabilitation by adults with hearing loss: an ecological model. PhD Thesis. Vancouver, Canada: University of British Columbia, 2002.

3. Engelund G. Time for hearing-recognising process for the individual. PhD Thesis. Eriksholm, Denmark: University of Copenhagen, 2006.

4. Manchaiah VKC, Stephens D, Meredith R. The patient journey of adults with hearing impairment: the patients' view. Clin Otol 2011;36:227-34.

5. Manchaiah VKC, Stephens D. The patient journey: living with hearing impairment. J Acad Rehab Audiol 2011;44:29-40.
6. Davis A, Smith P, Ferguson M, et al. Acceptability, benefit and costs of early screening for hearing disability: a study of potential screening tests and models. Health Tech Assess 2007;11:1-294.

7. Manchaiah VKC, Danermark B, Rönnberg J, et al. Importance of 'process evaluation': examples from studies on hearing impairment. Submitted.

8. Carson AJ. "What brings you here today?" The role of self-assessment in help-seeking for age-related hearing loss. J Aging Stud 2005;19:185-200.

9. Martin FN, Krall L, O'Neal J. The diagnosis of acquired hearing loss. ASHA 1989;31:47-50.

10. Light KJ, Looi V. Reactions to the diagnosis of a progressive hearing loss in adults. J Acad Rehab Audiol 2011;84:53-84.

11. Meyer C, Hickson L, Khan A, et al. Investigations of the actions taken by adults who failed a telephone-based hearing screen. Ear Hear 2011;32:720-31.

12. Brookes DN. The effect of attitude on benefit obtained from hearing aids. Br J Audiol 1989;23:3-11.

13. Jerram JCK, Purdy SC. Technology, expectations, and adjustment to of hearing loss: predictors of hearing aid outcome. J Am Acad Audiol 2001;12:64-79.

14. Hallberg L, Carlsson S. A qualitative study of strategies for managing a hearing impairment. Br J Audiol 1991;25:201-11.

15. Andersson G, Melin L, Lindberg $P$, et al. Development of a short scale for self-assessment of experiences of hearing impairment: the hearing coping assessment. Scand Audiol 1995;24:147-154.

16. Andersson $G$, Melin L, Lindberg $P$, et al. Elderly hearing impaired persons' coping behavior. Int J Behav Med 1996;3:303-20.

17. Knudsen LV, Oberg M, Nielsen C, et al. Factor influencing help seeking, hearing aid uptake, hearing aid use and satisfaction with hearing aids: a review of the literature. Trends Amplif 2010;14:127-54.

18. Hayes SC, Strosahl KD, Wilson KG. Acceptance and commitment therapy an experiential approach to behavior change. New York: The Guilford Press, 1999.

19. Wentzel KR, Caldwell K. Friendships, peer acceptance, and group membership: relations to academic achievement in middle school. Child Dev 1997;68:1198-209.

20. Li L, Moore D. Acceptance of disability and its correlates. J Soc Psychol 1998;138:13-25.

21. Stuifbergen A, Becker $\mathrm{H}$, Blozis $\mathrm{S}$, et al. Conceptualization and development of the chronic health conditions scale. Issues Ment Health Nurs 2008;29:101-14

22. Andersson $\mathrm{G}$, Willebrand $\mathrm{M}$. What is coping? A critical review of the construct and its application in audiology. Int $J$ Audiol 2003;42: S97-103.

23. Karekla M, Panayiotou G. Coping and experiential avoidance: unique or overlapping constructs? J Behav Ther Exp Psy 2011;42:163-70.

24. Goffman E. Stigma: notes on management of spoiled identity englewood cliffs. NJ: Prentice Hall, 1963.

25. Hétu R. The stigma attached to hearing impairment. Scand Audio 1996;25(Suppl 43):12-24.

26. Danermark B, Gellerstedt LC. Hearing impairment, psychosocial work environment and health. Int J Audiol 2004;43:383-9.

27. Linkowski DC. A scale to measure acceptance of disability. Rehab Couns Bull 1971;14:236-44.

28. McCracken LM, Vowles KE, Eccleston C. Acceptance of chronic pain: component analysis and revised assessment method. Pain 2004;107:159-66

29. McCracken LM, Vowles KE. Acceptence of chronic pain. Curr Pain Headache Rep 2006;10:90-4.

30. Westin V, Hayes SC, Andersson G. Is it the sound or your relationship to it? The role of acceptance in predicting tinnitus impact. Behav Res Ther 2008;46:1259-65.

31. Demorset ME, Erdman SA. Development of the communication profile for the hearing impaired. J Speech Lang Hear Res 1987;52:129-43.

32. Gomez RG, Mandey SF. Coping-with-hearingg-loss model for older adults. J Gerontol: Psychol Sci 2001;56:223-5.

33. Manchaiah VKC, Stephens D, Andersson G, et al. Use of the 'patient journey' model in the internet-based pre-fitting counseling of a person with hearing disability: study protocol for a randomized controlled trial. Trials 2013;14:25.

34. Manchaiah VKC, Rönnberg J, Andersson G, et al. Use of the 'patient journey' model in the internet-based pre-fitting counseling of a person with hearing disability: Lessons from a failed clinical trial. Submitted

35. Hayes SC, Strosahl KD, Wilson KG, et al. Measuring experiential avoidance: a preliminary test of a working model. Psychol Rec 2004;54:553-78. 
36. Noble $\mathrm{W}$, Tyler $\mathrm{R}$, Dunn $\mathrm{C}$, et al. Hearing handicap ratings among different profiles of adult cochlear implant users. Ear Hear 2008;29:112-20.

37. Herrmann C. International experience with the hospital anxiety and depression scale-a review of validation data and clinical results. J Psychosom Res 1997;42:17-41.

38. Zigmond AS, Snaith RP. The hospital anxiety and depression scale. Acta Psychatr Scand 1983;67:361-70.

39. Andersson G, Kaldo-Sandström V, Ström L, et al. Internet administration of the hospital anxiety and depression scale (HADS) in a sample of tinnitus patients. J Psychosom Res 2003;55:259-62.

40. McConnaughy EN, Prochaska JO, Velicer WF. Stages of change in psychotherapy: measurement and sample profiles. Psychother Theor Res Prac 1983;20:368-75.

41. DiClemente CC, Prochaska J. Toward a comprehensive, transtheoretical model of change. Miller \& Heather, ed. Treating addictive behaviors. 2nd edn. New York: Plenum Press, 1989: 3-24.

42. Laplante-Lévesque A, Hickson L, Worral L. Stages of change in adults with acquired hearing impairment seeking help for the first time: application of the transtheoretical model in audiologic rehabilitation. Ear Hear 2013;34:447-57.

43. Kline P. An easy guide to factor analysis. London: Routledge, 1994.

44. Garson GD. Reliability analysis statnotes: topics in multivariate analysis. http://faculty.chass.ncsu.edu/garson/PA765/reliability.htm (accessed 17 Dec 2012)

45. Kaiser HF. The applications of electronic computers to factor analysis. Edu Psychol Meas 1960;20:141-51.

46. Cattell RB. The scree test for the number of factors. Multivar Behav Res 1966;1:245-76.

47. Costello AB, Osborne JW. Best practice in exploratory factor analysis: four recommendations for getting the most from your analysis. Pract assess, Res Eval 2005;10:1-9. http://pareonline.net pdf/v10n7.pdf

48. Westin VZ, Schulin M, Hesser H, et al. Acceptance and commitment therapy versus tinnitus retraining therapy in the treatment of tinnitus: a randomised controlled trial. Behav Res Ther 2011;49:737-47.

49. Hesser $\mathrm{H}$, Gustafsson $\mathrm{T}$, Lundén $\mathrm{C}$, et al. A randomized controlled trial of Internet-delivered cognitive behavior therapy and acceptance and commitment therapy in the treatment of tinnitus. J Consult Clin Psychol 2012;80:649-61.

50. Hayes SC, Villatte M, Levin M, et al. Open, aware, and active: contextual approaches as an emerging trend in the behavioral and cognitive therapies. Annu Rev Clin Psychol 2011;7:141-68.

51. Bond FW, Bunce D. The role of acceptance and job control in mental health, job satisfaction, and work performance. J Appl Psychol 2003;88:1057-67.

52. Hayes SC, Stroshal K, Wilson KG, et al. Measuring experientia avoidance: a preliminary test of a working model. Psychol Rec 2004;54:553-78.

53. Bond FW, Lloyd J, Guenole N. The work-related acceptance and action questionnaire (WAAQ): initial psychometric findings and their implications for measuring psychological flexibility in specific contexts. J Occup Organ Psychol 2012:1-25.

54. Hayes SC, Strosahl KD, Wilson KG. Acceptance and commitment therapy: the process and practice of mindful change, 2nd edn. New York: Guilford Press, 2012.

55. McCracken LM. Learning to live with the pain: acceptance of pain predicts adjustment in persons with chronic pain. Pain 1998;74:21-7.

56. Ruiz FJ. A review of acceptance and commitment therapy (ACT) empirical evidence: correlational, experimental psychopathology, component and outcome studies. Int J Psych Psychol Ther 2010;10:125-62.

57. Swan IRC, Gatehouse S. Factors influencing consultation for management of hearing disability. Brit J Audiol 1990;24:155-60.

58. Whitehead LC. Methodological and ethical issues in internet-mediated research in the field of health: an integrated review of the literature. Soc Sci Med 2007;65:782-91.

59. Glasgow RE, Nelson CC, Kearney KA. Reach, engagement, and retention in an internet-based weight loss program in a multi-site randomized controlled trial. J Med Internet Res 2007;9:e11.

60. Thorén E, Andersson G, Lunner T. The use of research questionnaires in hearing impaired adults: online vs paper-and-pencil administration. BMC Ear Nose Throat Disord 2012;12:1-12.

61. Buchanan T. Online assessment: desirable or dangerous? Prof Psychol-Res Pr 2002;33:148-54. 